to additional tests on resistance mutations with AmpliSens ${ }^{\circledR}$ M.genitalium-ML/FQ-Resist-FL (Central Research Institute of Epidemiology, Russia) is shown.

Approach We included 13 clinical cases of M. genitalium infection with prevalence of men practicing sex with men patients. Clinical samples were collected from urethra, oropharynx and rectum. Extraction was performed using DNAsorb-AM (AmpliSens, Russia). Identification of M. genitalium was performed using «AmpliSens ${ }^{\circledR}$ N.gonorrhoeae/C.trachomatis/M.genitalium/T.vaginalis-MULTIPRIME-FRT» (CRIE, Russia). Resistance-associated mutations were detected using AmpliSens $^{\circledR}$ M.genitalium-ML/FQ-Resist-FL (CRIE, Russia) with validation against Sanger sequencing.

Impact M. genitalium was detected in all 13 studied cases of which $46.1 \%(n=6)$ in urethral swabs, 38.5\% $(n=5)$ in rectal swabs, and two with multiple sites of infection, including oropharynx. Most patients did not show clinical signs of M. genitalium infection while others reported urethral itching (7.7\%), anal region pain (7.7\%), and rectum bloody discharge after defecation (7.7\%). Resistance-associated mutations were detected in ten $(76.9 \%)$ cases, while seven of them $(70 \%)$ contained both $23 \mathrm{~S}$ rRNA and parC mutations. Administrated treatment was chosen according to qPCR resistance mutations test and consisted of Doxycycline or Minocycline $(100 \mathrm{mg}$ orally 2 times a day for 10 days) for resistant M. genitalium, and Josamycin (500 mg orally 3 times a day for 10 day) for non-resistant $M$. genitalium. In all cases infection was completely eradicated after treatment.

Innovation and Significance Determination of M. genitalium resistance associated mutations recommended both in European guideline on Mycoplasma genitalium infections and in BASHH UK national guideline. With high prevalence of both macrolides and fluoroquinolones associated mutations in our study authors imply that application of AmpliSens ${ }^{\circledR}$ M.genitalium-ML/FQ-Resist-FL kit with simultaneous detection of both 23 rRNA and parC genes mutations is a helpful tool to a clinical practice.

\section{P084 SCREENING OF ANORECTAL AND OROPHARYNGEAL SAMPLES FAILS TO DETECT BACTERIOPHAGES INFECTING NEISSERIA GONORRHOEAE}

1J Laumen*, 'S Abdellati, 'SS Manoharan-Basil, ${ }^{1,2} \mathrm{C}$ Van Dijck, ${ }^{1} \mathrm{D}$ Van den Bossche, ${ }^{1} \mathrm{D}$ Baetselier, ${ }^{1} \mathrm{~T}$ De Block, ${ }^{2} \mathrm{~S}$ Malhotra-Kumar, ${ }^{1,3} \mathrm{P}$ Soentjens, ${ }^{4} \mathrm{~J}$ Pirnay, ${ }^{1,5} \mathrm{C}$ Kenyon, ${ }^{4,6} \mathrm{M}$ Merabishvili. ${ }^{1}$ Institute of Tropical Medicine, Department of Clinical Sciences, Antwerp, Belgium; 'Laboratory of Medical Microbiology, Vaccine and Infectious disease Institute, University of Antwerp, Antwerp, Belgium; ${ }^{3}$ Center for Infectious diseases, Queen Astrid Military Hospital, Neder-over-Heembeek, Brussels, Belgium; ${ }^{4}$ Laboratory for Molecular and Cellular Technology (LabMCT), Queen Astrid Military Hospital, Neder-over-Heembeek, Brussels, Belgium; ${ }^{5}$ Department of Medicine, University of Cape Town, Cape Town, South Africa; ${ }^{6}$ Microbiology and Virology (EIBMV), Eliava Institute of Bacteriophage, Tbilisi, Georgia

10.1136/sextrans-2021-sti.216

Background There are real concerns that Neisseria gonorrhoeae may become untreatable in the near future due to the rapid emergence of antimicrobial resistance. Alternative therapies are thus urgently required. Bacteriophages active against N. gonorrhoeae could play an important role as an antibioticsparing therapy which may even reduce the selection pressure for the emergence of resistance against conventional antimicrobials. Furthermore, they could be used in combination with antimicrobial agents to treat multi-resistant N. gonorrhoeae.
To the best of our knowledge, no phages active against $\mathrm{N}$. gonorrhoeae have ever been found.

Methods The aim of this study was to screen for bacteriophages able to lyze N. gonorrhoeae in 194 oropharyngeal and 18 anorectal ESwabsTM of 74 men who have sex with men attending a sexual health clinic in Antwerp, Belgium. ESwabsTM were enriched for bacteriophages using 11 different clinical N. gonorrhoeae strains. The spot test method was used as an initial indicator test to screen for the presence of phages by measuring lytic activity.

Results Multiple clear zones were detected as a result of antibacterial activity, but none of the confluent lysis zones could be replicated through further propagation. We screened 212 swabs but were unable to identify an anti-gonococcal bacteriophage.

Conclusion This is the first report of a large-scale screening that systematically searched for anti-gonococcal phages directly from clinical swabs. A strictly virulent $\mathrm{N}$. gonorrhoeae phage would be of considerable utility in the fight against multi resistant N. gonorrhoeae infections. Further studies may derive more success by screening for phages at other anatomical sites (e.g., stool samples, urine) or in environmental settings (e.g., toilet sewage water of sex clubs or sexually transmitted infection clinics).

\section{P087 SPATIAL AND TEMPORAL EPIDEMIOLOGY OF INFECTIOUS SYPHILIS IN VICTORIA, AUSTRALIA, 2015- 2018}

${ }^{1}$ E Aung ${ }^{*},{ }^{1,2} \mathrm{M}$ Chen, ${ }^{1,2} \mathrm{C}$ Fairley, ${ }^{3} \mathrm{~N}$ Higgins, ${ }^{4} \mathrm{D}$ Williamson, ${ }^{5} \mathrm{~J}$ Tomnay, ${ }^{1} \mathrm{~K}$ Cook, ${ }^{1} \mathrm{~J}$ Peel, ${ }^{1} \mathrm{~V}$ Dharmakulasinghe, ${ }^{3} \mathrm{C}$ Alpren, ${ }^{1,2,6} \mathrm{E}$ Chow. ${ }^{1}$ Melbourne Sexual Health Centre, Melbourne, Australia; ${ }^{2}$ Central Clinical School, Faculty of Medicine, Nursing and Health Sciences, Monash University, Melbourne, Australia; ${ }^{3}$ Department of Health and Human Services, Melbourne, Australia; ${ }^{4}$ Microbiological Diagnostic Unit Public Health Laboratory, Department of Microbiology and Immunology, The University of Melbourne at The Doherty Institute for Infection and Immunity, Melbourne, Australia; ${ }^{5}$ Centre for Excellence in Rural Sexual Health, Melbourne Medical School, The University of Melbourne, Melbourne, Australia; ${ }^{6}$ Centre for Epidemiology and Biostatistics, Melbourne School of Population and Global Health, The University of Melbourne, Melbourne, Australia

10.1136/sextrans-2021-sti.217

Background The study aims to examine the trends of syphilis infection in Victoria and the characteristics of notified cases of syphilis among different population groups stratified by risk and in different geographical distributions.

Methods We analysed the demographic characteristics, notification trends and geographical distribution of notified cases of syphilis in Victoria between 2015 and 2018.

Infectious syphilis cases were categorised into four population groups: men who have sex with men (MSM), men who have sex with women (heterosexual males), females, and men who have sex with men and women (bisexual males). We examined the staging of syphilis, geographic location by residence of cases, HIV status, reasons for testing, and notifying source.

Results Of the 4,808 notified infectious syphilis cases, there were 3,801 (64\%) MSM, 593 (12\%) heterosexual males, 465 (10\%) females, and $118(2 \%)$ bisexual males. Females $(219 \%$ increase, ptrend $<0.001)$ and bisexual males $(220 \%$ increase, ptrend $=0.004$ ) had the greatest increase in the number of cases, followed by heterosexual males $(129 \%$ increase, ptrend $<0.001)$ and MSM $(21 \%$ increase, ptrend $<0.001)$. Geographical mapping showed the majority of the syphilis cases in 